\title{
Joint force of individual, hospital, government: Simulation of the spread of 2019 novel coronavirus by multi-agents complex adaptive system
}

Weiqiong Zhong ( $\sim$ zhongweiqiong2016@126.com )

MLR Key Laboratory of Metallogeny and Mineral Assessment, Institute of Mineral Resources, Chinese Academy of Geological Sciences

Tianxiu Wang Institute for Health Research and Policy, University of Illinois at Chicago

\section{Research Article}

Keywords: COVID-19, multi-agents complex adaptive system model, scenario simulation

Posted Date: April 22nd, 2020

DOl: https://doi.org/10.21203/rs.3.rs-24314/v1

License: (c) (i) This work is licensed under a Creative Commons Attribution 4.0 International License.

Read Full License 


\section{Abstract}

Early forecasting is important for health officials and decision-makers to respond to public health emergencies such as the 2019 novel coronavirus (COVID-19) outbreak. The spread of epidemic is mainly impacted by the interaction among agents, i.e., individual, hospital and government. In an effort to efficiently mitigate the impact of the virus, this original study designed a multi-agents complex adaptive system model of COVID-19 to detect the trend of the spread in 8 countries by simulating the adaptation and interaction among the agents. The results show that there should be 127,726 infections in China, 12,000 in South Korea, 729,377 in the USA, 176,623 in Italy, 194,359 in Spain, 165,122 in Germany, 177,462 in France and 149,540 in UK. It is impossible to screen and report all the cases, and the detection rate may be $82 \%$ in Italy, $65 \%$ in China, $55 \%$ in the USA, and $41 \%$ in UK on April 8,2020 . Scenario simulation results imply that action time is the most important factor in containing the spread of epidemic. If China had locked down Wuhan city five days later, the total infection would be tripled. The forces of individual, hospital and government should be united in fighting the virus.

\section{Main Text}

Since December 2019, an unexplained pneumonia has been reported in Wuhan city, Hubei Province, China, and then spreads to other areas in Mainland China and has now been detected in more than 200 locations over the world. As of April 8, 2020, there were a total of 1,366,689 confirmed cases among which 83,249 were located in Mainland China. Scholars around the world have carried out a lot of researches, such as clinical features 1,2, genomic characteristics3,4, epidemiologic characteristics2,5 and spread risks6,7 analyses. These studies provide valuable reference information for prevention and containment.

It is important for decision-makers to assess the infection situation and the effect of prevention and control in advance. Given the fact that local emergence may lead to regional outbreaks or global pandemics, the economic impacts can be devastating8. Timely forecast can help them deploy medical resources and rescue supplies properly. The chaos that emerged in the early days of Wuhan city's lockdown reflects the lack of early forecasting. Based on the ascertainment rate of infection in Japanese citizens evacuated from Wuhan some scholars estimated that the ascertainment rate of infection was around $9.2 \%$ and incidence of infection in Wuhan can be 20,767 as of January 29,20209 . An exponential growth model was adopted to estimate the epidemic curve of COVID-19 cases in mainland China and found that there may be 469 unreported cases from 1 to January 15, 2020. The reporting rate after January 17,2020 was likely to have increased 21 -fold10. A simple disease-transmission model of COVID-19 was established and found that reported number of cases remain lower than modeled estimates, but ascertainment is increasing over time11. A susceptible exposed infectious recovered metapopulation model was used to simulate the epidemics across China, and estimated that that 75,815 individuals have been infected in Wuhan as of January 25, 202012. Using the generalized logistic growth model, the Richards growth model, and the sub-epidemic wave model, Roosa et al. predicted that an 
average total cumulative case was between 37,415 and 38,028 in Hubei and 11,588-13,499 in other provinces by February 24, 202013.

These studies provide estimation results for COVID-19 in China. However, the forecasts made on different date vary widely. This is because the process of epidemic is full of dynamic and uncertainty, and traditional susceptible exposed infectious recovered model doesn't account for the adapting behavior of individuals, the changing of the medical resources and governments control power, or the interaction between them14. Complex adaptive system is a model to simulate the complex and changing reality 15 . It is an alternative approach to better reflect the adapting and changing nature of epidemic16. Many factors and multiple agents play different roles in the whole process which make it complex and adaptive17,18. Thus a multi-agents complex adaptive system model of epidemic is needed for the estimation19.

The aim of this study is to simulate the spread of COVID-19 using a multi-agents complex adaptive system model we originally designed, to reveal the roles of individual, hospital, and government in the prevention and control, to answer the questions about the time and number of peak infections, and to provide information for outbreak prevention and control policies in countries around the world. The advantage of this model is simulating the reality of the process of epidemic incorporating two key features that are different from the previous studies: dynamic and adaptive. In our model, three agents which play the key roles are selected: individual, hospital, and government. The dynamic is reflected by the interaction and adaptation between agents, and parameters such as isolation rate and spread rate are adjusted with time. The adaptive is reflected by assigning values to parameters in a fixed interval, such as reproduction number, incubation period, recover or death period for each individual. In this study, 8 countries were selected as research objects: China, South Korea, the USA, Italy, Spain, Germany, France, and UK. The total infections, the peak of illness, and the detection rate of illness are discussed. Three scenarios are set up to simulate the trend of spread under different control times for China and South Korea.

This original study addresses key issues in public health emergency of epidemic: how many individuals have been or will be infected, are there sufficient medical resources, and what is the impact of a prevention policy? These questions can be answered by the simulation of multi-agents complex adaptive system that models the adaptation and interaction among agents, i.e., individual, hospital, and government. The prediction of time and number of peak infections provide reference for the development of prevention policy and deployment of medical resources for the governments and also alarm the public.

\section{Interaction among individual, hospital and government}

The multi-agents complex adaptive system model of COVID-19 consists of agents, the adaptability of the agents, and the interaction among agents. Agents in this study are individual, hospital and government (please see Figure 1). Individuals are the objects of virus infection that needs protection and treatment and additionally, the behaviors of individuals are affected by other agents; hospitals (including community medical services) provide medical care and isolation to the individuals, and in the meanwhile, 
the expansion of medical resources depends on the deployment from the government; government implements prevention and control measures that affect the activities of individuals and hospitals.

\section{Simulation results}

Parameters of China are in Extended Data Table 1-4. The simulation results for Mainland China are shown in Figure 2. The total infection in Mainland China is 127,726 , among which 109,908 are located inside Hubei and 17,818 are located in other areas outside Hubei. The peak time of accumulative cases is March 17, 2020. The peak time of new added cases is February 3, 2020 with a total of 6,474 new cases, among which 5,247 are located inside Hubei and 1,227 are located outside Hubei. The simulation result is plotted in Figure 5. The fluctuation of the simulated cumulative infections is closely related to the sufficiency of medical care resources and the implementation of prevention and control. The lockdown of Wuhan city on January 23, 2020 and soon afterwards the lockdown of other cities lead to the inflection point of the curve. The building of Fire mountain hospital and Thunder mountain hospital, as well as the building of compartment hospitals provide more than 30,000 beds for medical treatment and isolation. Thus on February 5, 2020 the infection was sharply reduced. Once being infected, the symptom will show after the incubation period of $3 \sim 14$ days, thus the curve of simulated accumulative cases is lagging behind the curve of accumulative infection.

The multi-agents complex adaptive system models for other countries are constructed separately according to the unique action of their governments, hospitals and individuals. Parameters of them are in Extended Data Table 6-19 and the simulation results are in Figure 3. If strict prevention and control were implemented on March 23, 2020, the total infection in the USA would be 729,377 . If strict prevention and control were implemented on March 20,2020, the total infection in Italy would be 176,623 . If strict prevention and control were implemented on March 15,2020, the total infection in Spain would be 194,359. If strict prevention and control were implemented on March 25, 2020, the total infection in Germany would be 165,122. If strict prevention and control were implemented on March 24, 2020, the total infection in France would be 177,462. If strict prevention and control were implemented on March 24,2020 , the total infection in UK would be 149,540 . Strict prevention and control means the large area lockdown is implemented by the government, the majority of illness is isolated by the hospital, and the individuals are aware of the dangerous and reduce their contacts.

\section{Detection rate}

The multi-agents complex adaptive system model of COVID-19 is simulating the natural development of an epidemic, that is, how many individuals will be infected and when they will show symptoms. Due to the high proportion of individuals with mild symptom, a large number of illnesses will not be tested and they cannot be included in the reported data. This study uses detection rate to measure the proportion of 
discovered illness. Detection rate is calculated by $\mathrm{dr}=\frac{N_{\text {con }}}{N_{\text {sim }}}$, where $N_{\text {con }}$ is actual data of accumulative confirmed cases and $N_{\text {sim }}$ is simulated results of cumulative illness.

The detection rate inside Hubei, outside Hubei and total in the Mainland China are shown in Figure 4. The detection rate inside Hubei is 18\% at the beginning. Between January 21, 2020 and January 27, 2020, the hospitals in Hubei are overloaded and the virus testing facility is insufficient, thus the detection rate decreased during this period. As 131 hospitals in Hubei are opened gradually to accept patients and 89 institutions in Hubei established the nucleic acid testing ability, the detection rate increased rapidly. It should be noted that on February 12, 2020 the detection rate in Hubei burst from 39\% to 54\%. The causation is that the official diagnostic criteria changed from nucleic acid test to clinical diagnosis paralleled to nucleic acid test. The confirmed cases in Hubei thus burst and then the detection rate increased to $62 \%$ on April 2, 2020.

The detection rate outside Hubei is lower than $10 \%$ at the beginning, due to the unawareness of the official agencies and individuals. However, after Nanshan Zhong issued warning on TV the prevention and control measures were implemented. Relatively fewer patients and adequate medical resources make it possible for health officials to find more infected person and trace their close contacts. The detection rate outside Hubei increased much faster than that in Hubei. At the end of January, the growth rate of detection rate outside Hubei declined. The detection rate outside Hubei increased to 83\% on April 2, 2020.

The detection rate in Mainland China is between the detection rate inside Hubei and outside Hubei, and is closer to the detection rate inside Hubei because more than $80 \%$ confirmed cases in Mainland China are located inside Hubei. The detection rate in Mainland China reached 65\% on April 2, 2020.

In the early stage, due to insufficient detection capabilities, inadequate hospital admissions, and delays for patients to go to hospital, the actual reported data was smaller than (or lagging behind) simulated data. However, as the medical resource support assembled and the detection capability increased, there is still a gap between the simulated results and the real-time data. The detection rate may keep growing in the rest period of the epidemic. However, there are four factors making it difficult to reach a detection rate of 100\%: (a) Asymptomatic self-recovery; (b) Symptomatic self-recovery; (c) Patients who have symptoms but cannot be diagnosed due to nucleic acid test errors; (d) Patients who have progressed rapidly and died before their diagnosis. Due to the existence of these four situations, there will always be a gap between the true number of infection this study tries to simulate and the official reported number of confirmed cases.

The detection rates of all the studied countries on April 8, 2020 are shown in Figure 5. South Korea may have the highest detection rate of $85 \%$, the detection rate of the USA is $55 \%$, and UK may have the lowest detection rate of $41 \%$.

\section{Scenarios}


The above results are simulated according to the real situation, for example, Wuhan city was locked down on January 23,2020 . What would happen if the lockdown be earlier or be later? This study designs three scenarios for China and South Korea and simulates the spread of the epidemic respectively (parameters are in Extended Data Table 5 and Table 7). The results are shown in Figure 6. If the lockdown of Wuhan were implemented on January 10, 2020, the total infection in Mainland China would be 4,327, if it were implemented on January 15,2020 , the total infection would be 26,112 , and if it were implemented on January 28,2020 , the total infection would be 477,370 . If South Korea had powerfully implemented the lockdown containment on February 20, the total infection would be around 4,000; if on February 25, the total infection would be around 12,000 (this may be the real situation); and if on March 1, the total infection would be around 24,000. The peak time of accumulative cases would change accordingly, and the total death would also change proportionally. The action time (i.e. starting time to prevent and control) plays the most important role in the whole process of the epidemic. Earlier action will cause much less illness and death.

\section{Conclusion}

Action time is the most important factor in containing the spread of epidemic. If the containment in Mainland China were implemented earlier on January 15,2020 , the infection would be $1 / 5$ of that on January 23. It may be no need to lock down the whole city. Controlling the hospitals, residential areas, and the close contacts were enough. If the containment were delayed to January 28, 2020, the total infection would be tripled. For other countries such as South Korea, if the powerful control was implemented 5 days later, the total infection would be doubled.

Individual, hospital and government all play important roles in the prevention and control. In our model, the setting of parameters such as isolation rate and spread rate has a great influence on the simulation results. Medical resources of the hospital mainly affect isolation rate. Lockdown and restriction policy from the government and the obedience of the individual mainly affect spread rate. Higher isolation rate, stricter policy and more cooperative individuals will reduce spread rate significantly.

This study provides a new approach of modeling COVID-19 by multi-agents complex adaptive system considering individual, hospital and government. It can help the decision-makers to pre-judge the development of the epidemic, implement the prevention and deploy the medical resources more scientifically. Restricted by the data availability, this study only chooses individual, hospital, and government as agents. If more agents such as research institution, news media, and transportation could be included, the multi-agents complex adaptive system model will be more effective. Other countries now facing the similar situation could also introduce this model to guide the implementation of epidemic prevention, and united the individual, hospital, and government in fighting the invasion of the virus.

\section{Methods}


Parameters for individual are population, incubation period, recover or death period, and reproduction number. Parameter for hospital is isolation rate which indicates the proportion of infected individuals that are isolated. It is related to the number of beds available for isolation and treatment. Parameter for government is spread rate which indicates the proportion of infected individuals that go out and spread the virus. It is related to the travel restriction and control of the infection source (please see Extended Data Figure 1). The parameters are evolving over time, interacting with each other, and influencing each other. If the government implements better prevention and control, the spread rate will decrease to a much lower level.

The reproduction number for every individual is 1 or 2 which means one infected person will spread the disease to 1 or 2 people in one day, but not every infected person go out and spread the disease. How many infected people go out and spread the disease depends on the isolation rate and spread rate. The isolation period (ip) is an integer randomly assigned from 3 to 14. The recover or death period (rp) is an integer randomly assigned form 7 to 20 . For every individual, once being infected at time t, he/she will be illed on day $\mathrm{t}+\mathrm{ip}$, and will be recover or die on day $\mathrm{t}+\mathrm{ip}+\mathrm{rp}$. Once recovered or dead, the individual will be removed from the population.

The infection and illness are calculated in a matrix (please see Extended Data Figure 2). Taking the population size of $n$, isolation rate (ir) $=0$, spread rate $(\mathrm{sr})=1$ as an example, the calculation of the infection and illness is shown in Figure 3. The numbers in red represent the individuals from 1 to $\mathrm{n}$. Individual No.1 is the original infected person. On day 1, the reproduction number of No.1 is 1, and No.1 spreads the disease to No.5, thus row 5 column 1 is marked by 1+ip+rp. On day 2, No.1 and No.5 are both infection resources. No.1 spreads the disease to No.2, and No.5 spreads the disease to No.7 and No.9, thus row 2 column 1, row 7 column 5, and row 9 column 5 are marked by $2+i p+r p$. Who will be infected is chosen randomly, and the infected individual may be repeated because in the reality the close contacts may also be overlapping. This process is repeated from day 1 to day t. The number of rows with marks at day $\mathrm{t}$ is the number of cumulative infection Clt. The individuals will be illed on day $\mathrm{Ti}, \mathrm{Ti}=\mathrm{t}+\mathrm{ip}$, and will recover or die on day $\mathrm{Tr}, \mathrm{Tr}=\mathrm{t}+\mathrm{ip}+\mathrm{rp}$. The parameter ip and $\mathrm{rp}$ are assigned separately for every individual. The number of illness on day $t$ is the number of individuals whose $\mathrm{Ti}=\mathrm{t}$. The number of recover or death on day $t$ is the number of individuals whose $\operatorname{Tr}=t$.

The number of source St means how many infected individuals will spread the disease on day t. The number of source is calculated by

$S_{t}=\left(S_{t-1}-C I_{t-1} * i r\right) * s r$

The model for mainland China is divided into two phases which simulated the process inside Hubei and outside Hubei separately (please see Extended Data Figure 3). The first phase is before the lockdown of Wuhan city, the virus is mainly spread inside Wuhan with the population of 14 million (the sum of permanent residents and floating population). After that, 5 million people in Wuhan traveled out for the Spring Festival, among which $75 \%$ went to other areas in Hubei, and $25 \%$ went to other provinces outside 
Hubei. Thus the second phase consists of two parts: inside Hubei with the population of 50 million, and outside Hubei with the population of 1000 million. The prevention and control effects are different inside and outside Hubei, thus the parameters are different. Once being constructed, the model was trained using the historical data. The parameters of isolation rate and spread rate are adjusted in the process of training and stopped when the curves of new illness are well fitted.

\section{Declarations}

\section{Acknowledgements}

This research is supported by grants from the National Natural Science Foundation of China (Grant No. 71991480, and No.71991485)

\section{Author Contributions}

These authors contributed equally to this work.

\section{Author Information}

Reprints and permissions information is available at www.nature.com/reprints. The authors declare no competing financial interests. Readers are welcome to comment on the online version of the paper. Correspondence and requests for materials should be addressed to Weiqiong Zhong. (zhongweiqiong2016@126.com)

\section{References}

1. Lee, W., Malik, N., Shi, F. \& Mucha, P. J. Social clustering in epidemic spread on coevolving networks. Phys. Rev. E 99, doi:10.1103/PhysRevE.99.062301 (2019).

2. Paina, \& Peters, D. H. Understanding pathways for scaling up health services through the lens of complex adaptive systems. Health Policy and Planning 27, 365-373, doi:10.1093/heapol/czr054 (2012).

3. Holland, J. H. Studying Complex Adaptive Systems. Journal of Systems Science and Complexity 19, 1-8, doi:10.1007/s11424-006-0001-z (2006).

4. Epstein, J. M. Modelling to contain pandemics. Nature 460, 687-687, doi:10.1038/460687a (2009).

5. Roosa, Y. L., R. Luo, A. Kirpich, R. Rothenberg, J.M. Hyman, P. Yan, G. Chowell,. Real-time forecasts of the COVID-19 epidemic in China from February 5th to February 24th, 2020. Infectious Disease Modelling 5, 256-263 (2020).

6. Wu, J. , Leung, K. \& Leung, G. M. Nowcasting and forecasting the potential domestic and international spread of the 2019-nCoV outbreak originating in Wuhan, China: a modelling study. Lancet (London, England), doi:10.1016/s0140-6736(20)30260-9 (2020). 
7. Tuite, R. \& Fisman, D. N. Reporting, Epidemic Growth, and Reproduction Numbers for the 2019 Novel Coronavirus (2019-nCoV) Epidemic. Annals of internal medicine, doi:10.7326/m20-0358 (2020).

8. Zhao, S. et al. Estimating the Unreported Number of Novel Coronavirus (2019-nCoV) Cases in China in the First Half of January 2020: A Data-Driven Modelling Analysis of the Early Outbreak. Journal of clinical medicine 9, doi:10.3390/jcm9020388 (2020).

9. Nishiura, H. et al. The Rate of Underascertainment of Novel Coronavirus (2019-nCoV) Infection: Estimation Using Japanese Passengers Data on Evacuation Flights. Journal of clinical medicine 9, doi:10.3390/jcm9020419 (2020).

10. Huang, C. et al. Clinical features of patients infected with 2019 novel coronavirus in Wuhan, China. Lancet (London, England) 395, 497-506, doi:10.1016/s0140-6736(20)30183-5 (2020).

11. Chen, N. et al. Epidemiological and clinical characteristics of 99 cases of 2019 novel coronavirus pneumonia in Wuhan, China: a descriptive Lancet (London, England) 395, 507-513, doi:10.1016/s0140-6736(20)30211-7 (2020).

12. Zhou, et al. A pneumonia outbreak associated with a new coronavirus of probable bat origin. Nature, doi:10.1038/s41586-020-2012-7 (2020).

13. Lu, R. et al. Genomic characterisation and epidemiology of 2019 novel coronavirus: implications for virus origins and receptor binding. Lancet (London, England) 395, 565-574, doi:10.1016/s01406736(20)30251-8 (2020).

14. Li, Q. et al. Early Transmission Dynamics in Wuhan, China, of Novel Coronavirus-Infected Pneumonia. The New England journal of medicine, doi:10.1056/NEJMoa2001316 (2020).

15. Du, et al. Risk for Transportation of 2019 Novel Coronavirus Disease from Wuhan to Other Cities in China. Emerging infectious diseases 26, doi:10.3201/eid2605.200146 (2020).

16. Tang, et al. Estimation of the Transmission Risk of the 2019-nCoV and Its Implication for Public Health Interventions. Journal of clinical medicine 9, doi:10.3390/jcm9020462 (2020).

17. Di Marco, M. et al. Opinion: Sustainable development must account for pandemic risk. Proceedings of the National Academy of Sciences of the United States of America 117, 3888-3892, doi:10.1073/pnas.2001655117 (2020).

18. Yang, , Xu, Z. P., Feng, J. W. \& Fu, X. C. Feedback pinning control of collective behaviors aroused by epidemic spread on complex networks. Chaos 29, doi:10.1063/1.5047653 (2019).

19. Scarpino, S. \& Petri, G. On the predictability of infectious disease outbreaks. Nat. Commun. 10, doi:10.1038/s41467-019-08616-0 (2019).

20. Novel Coronavirus Pneumonia Emergency Response Epidemiology, The epidemiological characteristics of an outbreak of 2019 novel coronavirus diseases (COVID-19) in China. Zhonghua liu xing bing xue za zhi = Zhonghua liuxingbingxue zazhi 41, 145-151, doi:10.3760/cma.j.issn.02546450.2020.02.003 (2020).

\section{Figures}




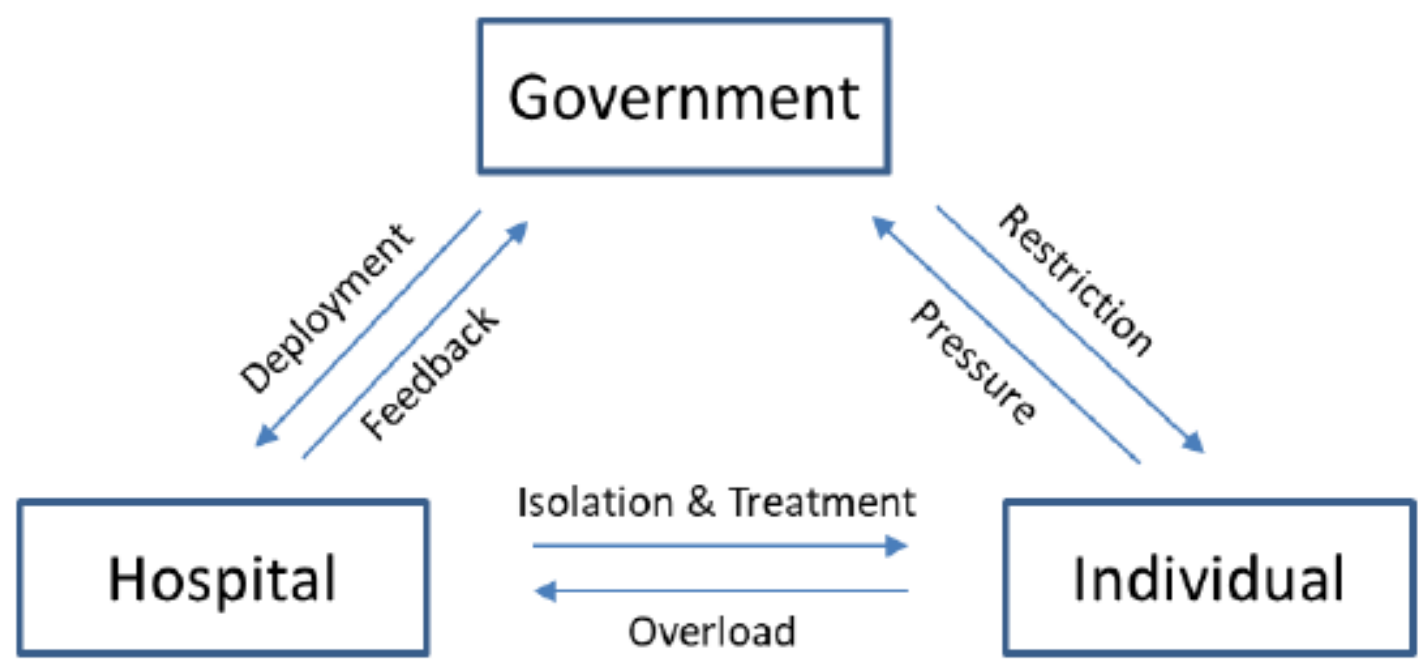

Figure 1

Interaction between the agents 

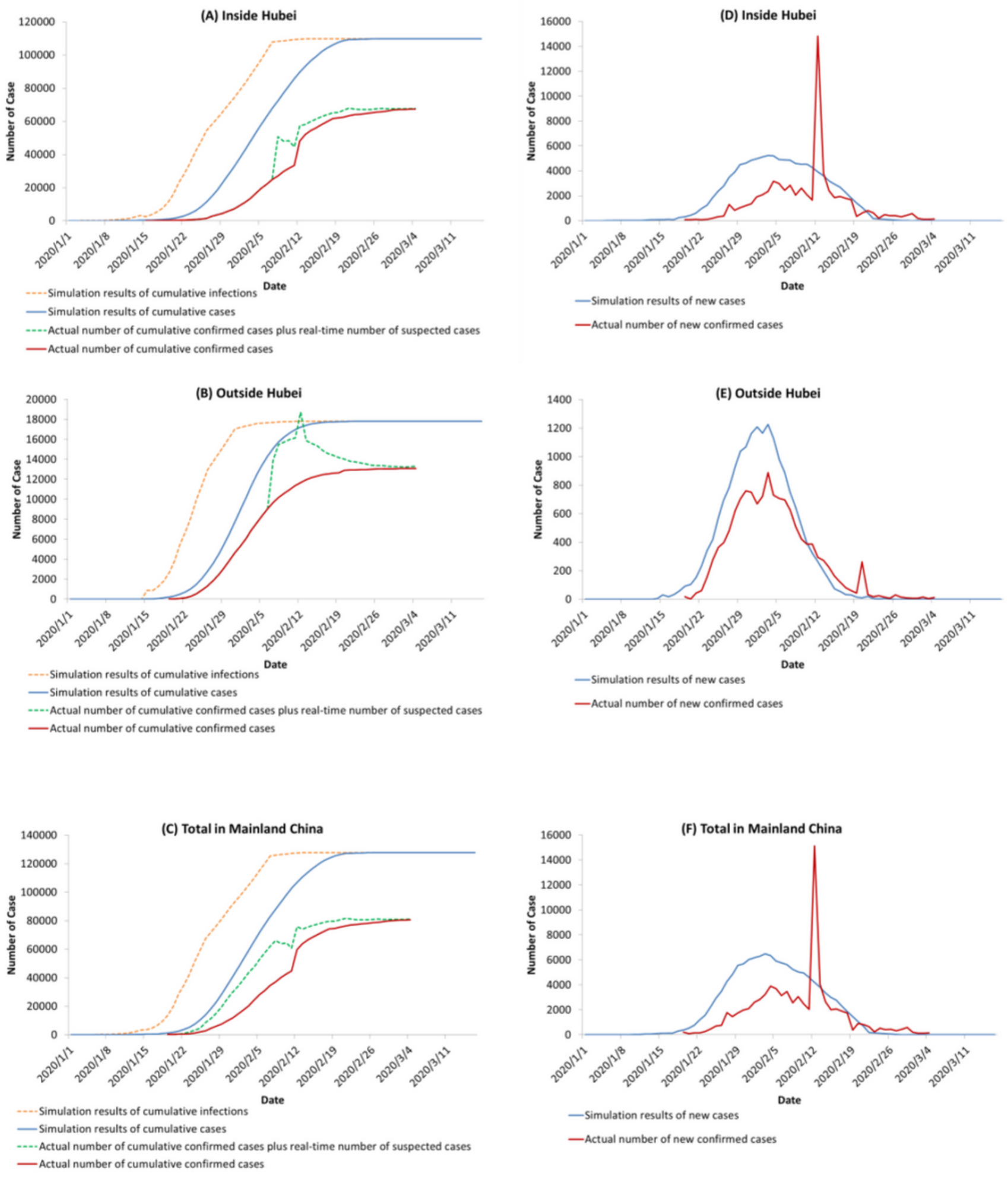

\section{Figure 2}

\section{Simulation results for Mainland China}



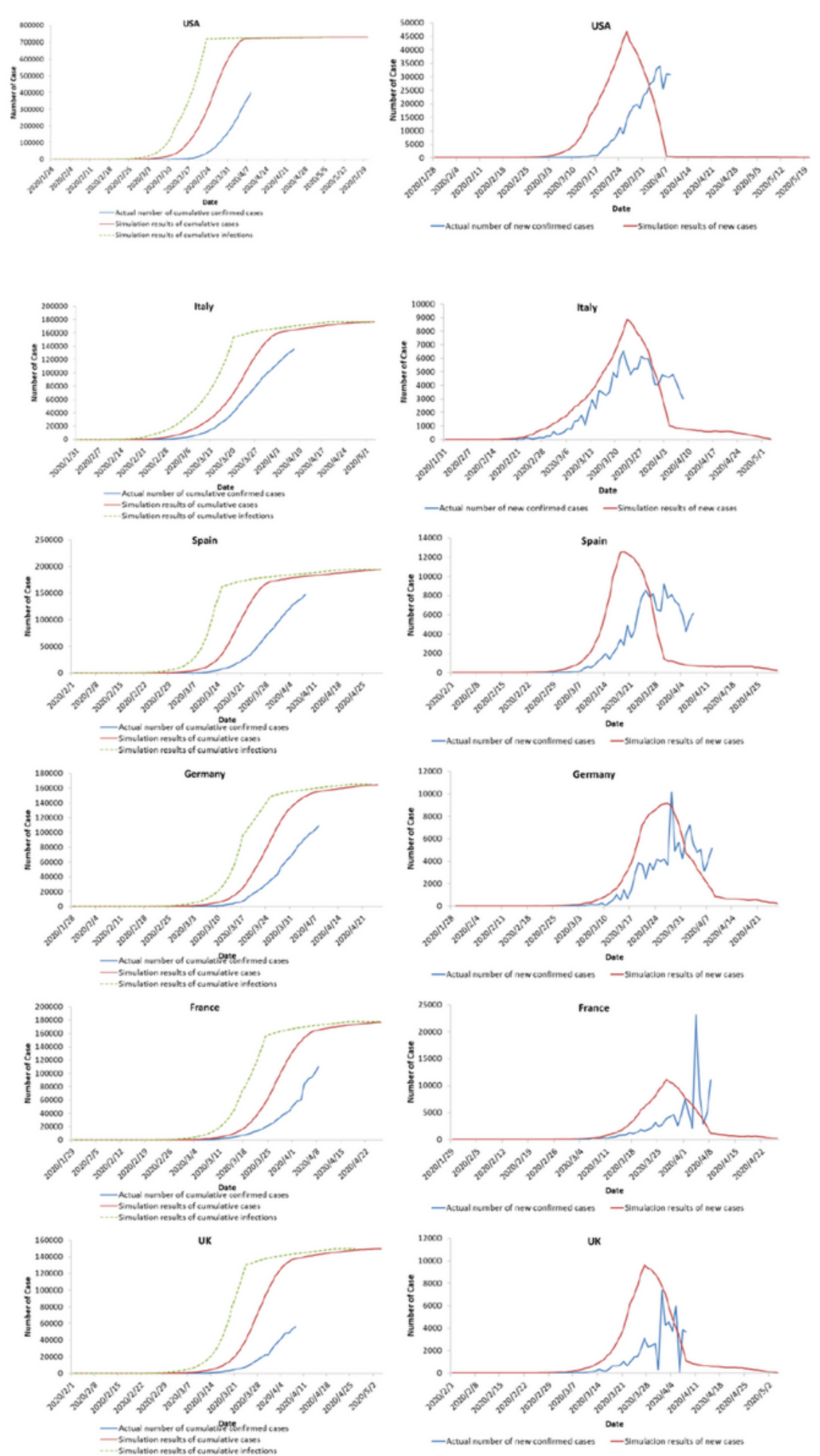

Figure 3

Simulation results for the USA, Italy, Spain, Germany, France, and UK

Page 12/14 


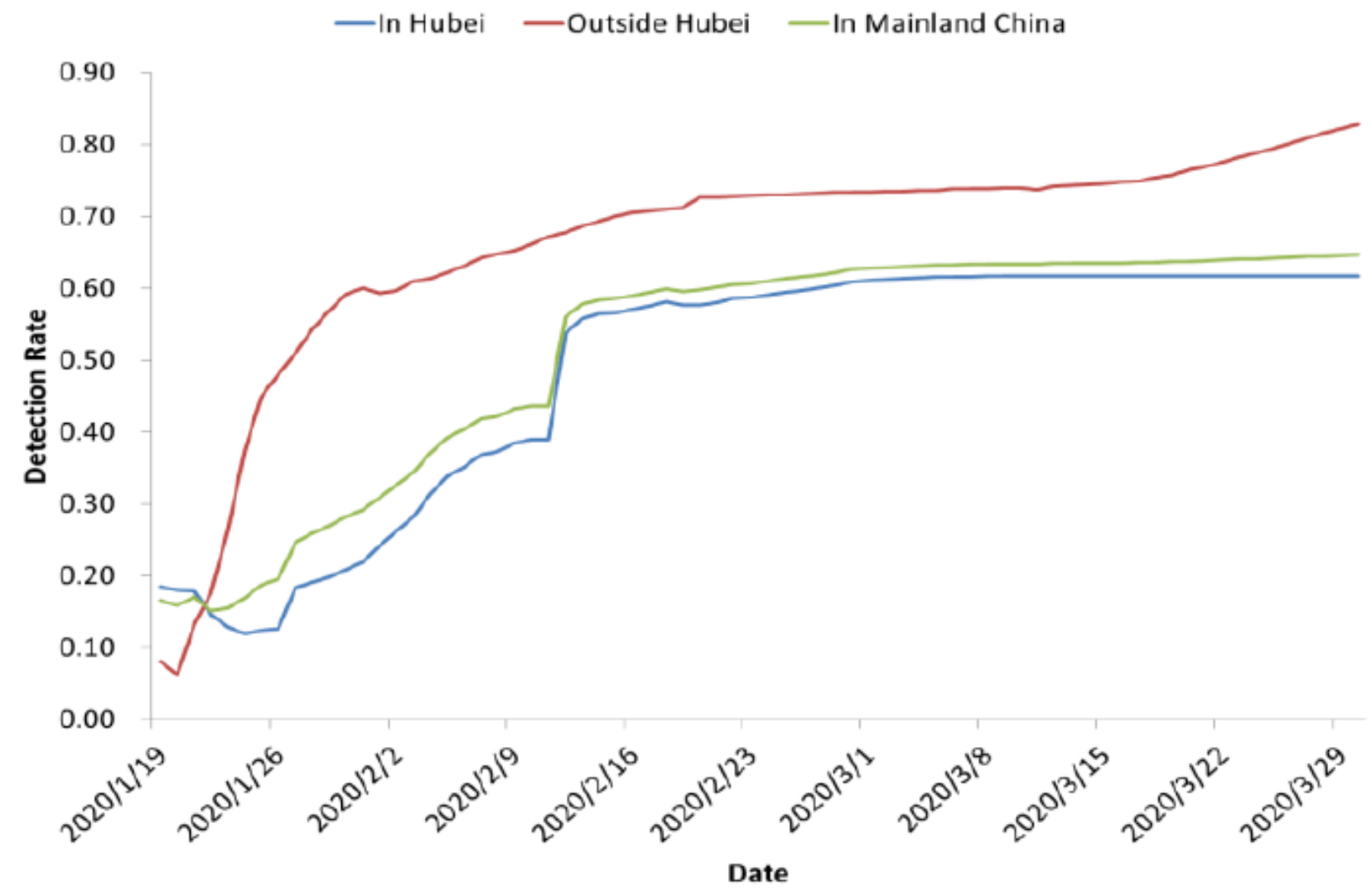

Figure 4

Detection rate inside Hubei, Outside Hubei and in Mainland China

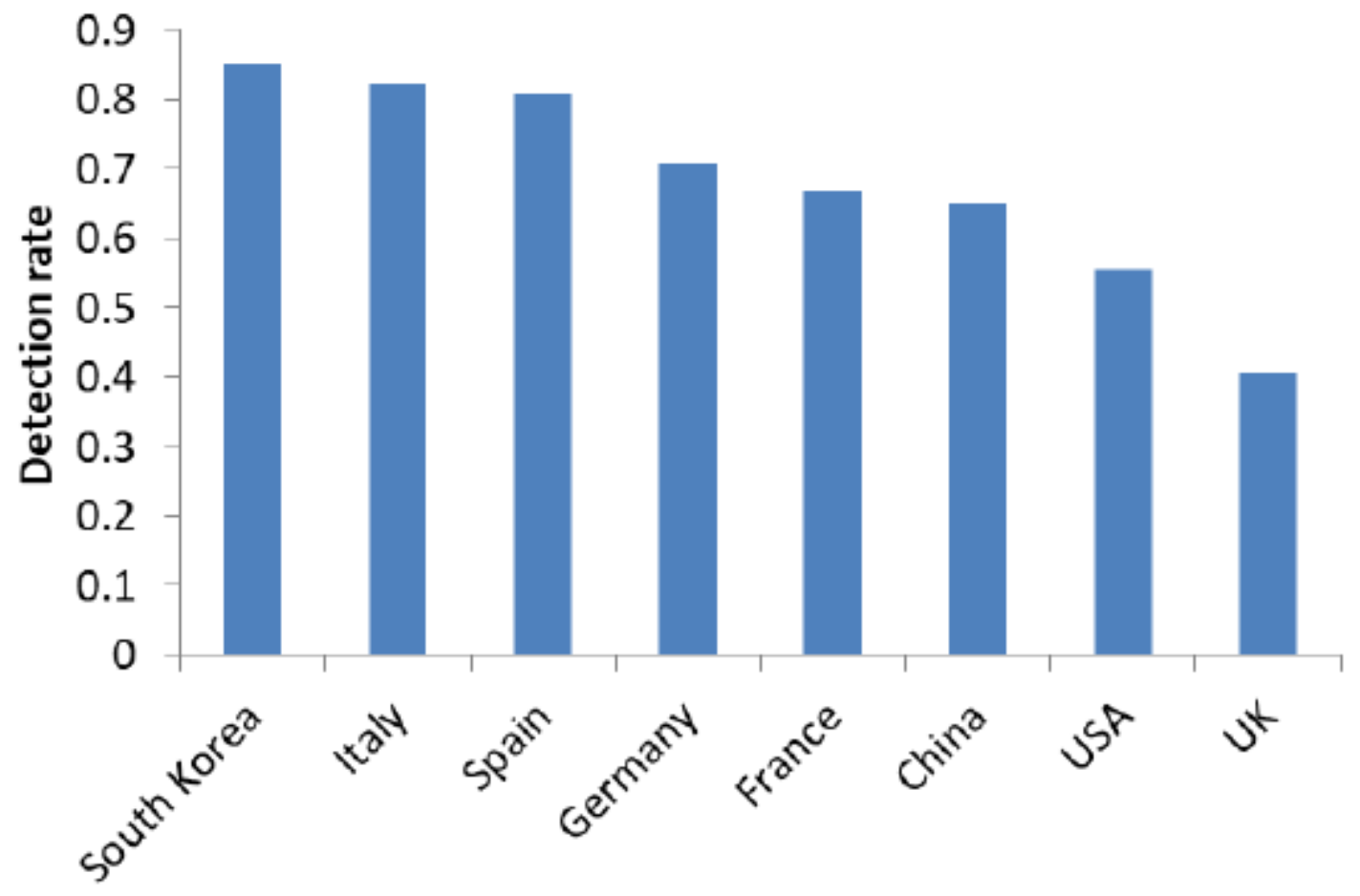

Figure 5 
Detection rates of the countries on April 8, 2020
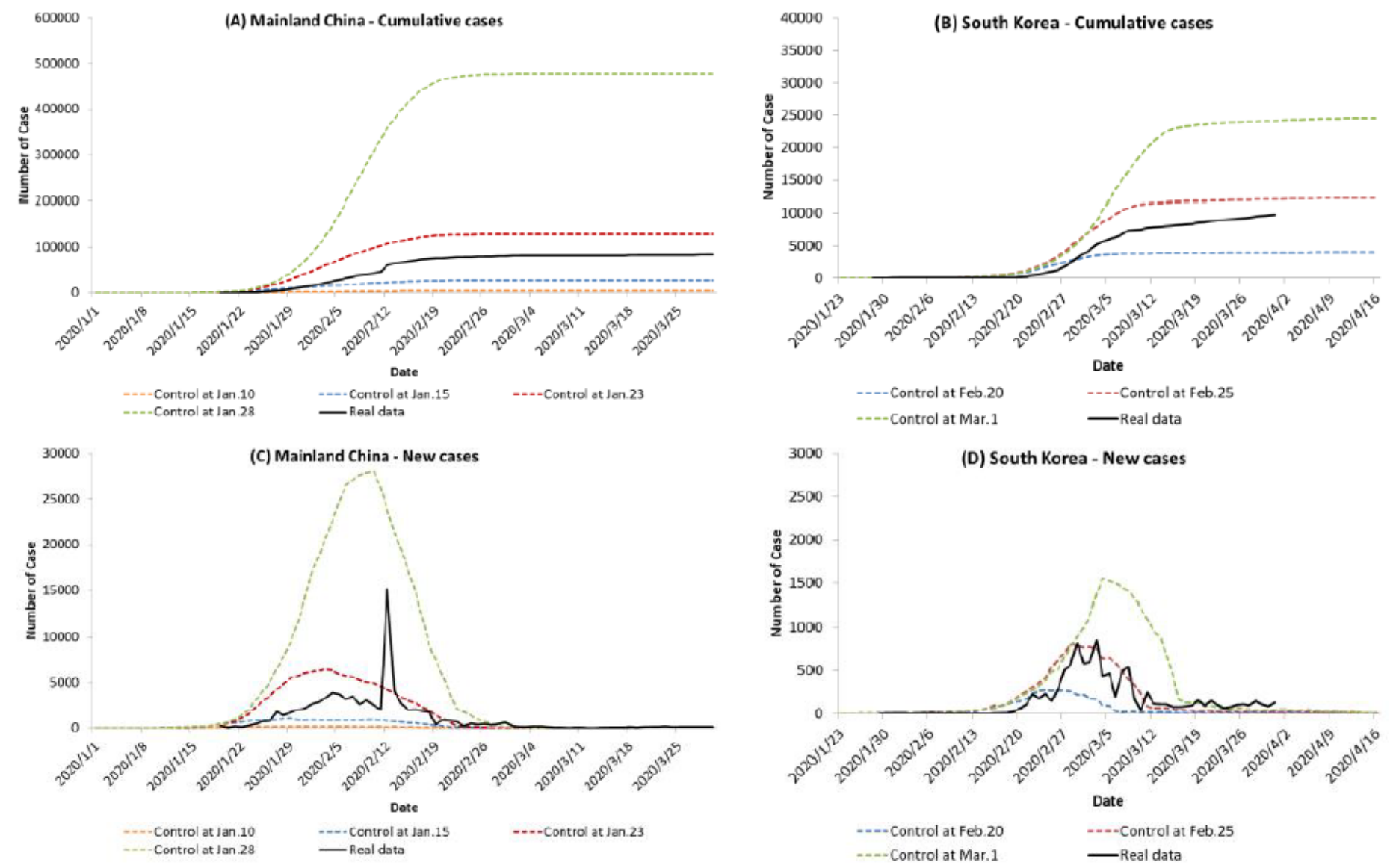

Figure 6

Scenarios for China and South Korea

\section{Supplementary Files}

This is a list of supplementary files associated with this preprint. Click to download.

- Extendeddata.pdf 